hep-ph/0503070

February 2005

To the memory of Leonid Avdeev

\title{
About higher order $\varepsilon$-expansion of some massive two- and three-loop master-integrals
}

\author{
M. Yu. Kalmykov \\ Department of Physics, \\ Baylor University, \\ One Bear Place, Box 97316 \\ Waco, TX 76798-7316 \\ Bogoliubov Laboratory of Theoretical Physics, \\ Joint Institute for Nuclear Research, \\ 141980 Dubna (Moscow Region), Russia
}

\begin{abstract}
For certain dimensionally-regulated massive two- and three-loop propagator-type diagrams the higher order $\varepsilon$-expansion is constructed.
\end{abstract}

PACS: 11.15.Bt; 02.30.Gp; 12.20.Ds; 12.38.Bx;

Keywords: Feynman diagrams; Master integrals; Higher order $\varepsilon$-expansion; 


\section{Introduction}

In the light of the recent progress in the four-loop calculations [1,2] one of the topical tasks is the construction of $\varepsilon$-expansion of one-, two-, and three-loop master-integrals of the existing packages like ONSHELL2 [3] or three-loop packages [4, 5, 6, 7] up to level sufficient for getting finite four-loop corrections in physical quantities. The all-order $\varepsilon$-expansion of the one-loop propagator diagram with arbitrary masses and external momentum, and the two-loop bubble diagram with arbitrary masses was constructed in $[8,9]$. The results are expressible in terms of Nielsen polylogarithms [10] only. The construction of the $\varepsilon$-expansion for diagrams relating to QED/QCD problems was investigated in $[11,12]$. The finite parts of the three-loop bubble integrals were presented in $[13,14,15]$. The higher order $\varepsilon$-expansion for some of the master-integrals from these packages was calculated in our previous papers ${ }^{1}$ $[9,16]$. Independently of analytical calculations, the numerical approach to the evaluation of the single scale diagrams has been developed in recent years [17]. Based on this technique, the high-precision numerical values of higher order coefficients of the $\varepsilon$-expansion for fourloop bubble integrals [18] and three-loop propagator type integrals on mass shell [19] have been calculated recently.

The present paper is devoted to the analytical calculation of the higher order terms of the $\varepsilon$-expansion for the scalar integrals shown in Fig. 1. At $M=m$ these integrals enter in packages ONSHELL2 [3] $\left(V_{1111}\right)$ and three-loop packages $[4,5]\left(D_{5}\right)$. The numerical values of higher order coefficients of the $\varepsilon$-expansion for diagram $V_{1111}$ and $D_{5}$ were calculated by Laporta in [17]. Another problem under consideration is further investigation and development of the "sixth root of unity" approach proposed by Broadhurst in [13] and developed in $[14,16,9,20,18]$. The main idea is that transcendental numbers occurring in the $\varepsilon$-expansion of single scale diagrams (diagrams with only one mass scale) are defined by the values of massive cuts. For the diagrams with zero-, one-, two-, and three- massive cuts, the set of transcendental numbers are related to generalized log-sine functions [21] or their generalization $[9,20]$ of special values of arguments $\{\pi, \pi / 2, \pi / 3,2 \pi / 3\}$. For diagrams with four massive cuts new constants, associated with the elliptic function [18] appeared.

We work in the dimensional regularization [22] with space-time dimension $n=4-2 \varepsilon$. In our normalization each loop is divided by $(4 \pi)^{2-\varepsilon} \Gamma(1+\varepsilon)$. We also use the following short notation for the auxiliary integrals appearing in our calculation:

$$
\begin{aligned}
J_{m_{1} m_{2} m_{3}} & =\left.\int \frac{d^{n} k_{1} d^{n} k_{2}}{\left[\left(k_{1}-p\right)^{2}+m_{1}^{2}\right]\left[k_{2}^{2}+m_{2}^{2}\right]\left[\left(k_{1}-k_{2}\right)^{2}+m_{3}^{3}\right]}\right|_{p^{2}=-m^{2}}, \\
V_{m_{1} m_{2} m_{3}} & =\int \frac{d^{n} k_{1} d^{n} k_{2}}{\left[k_{1}^{2}+m_{1}^{2}\right]\left[k_{2}^{2}+m_{2}^{2}\right]\left[\left(k_{1}-k_{2}\right)^{2}+m_{3}^{3}\right]}, \\
B_{0}\left(m_{1}, m_{2}, m_{3}\right) & =\left.\int \frac{d^{n} k_{1}}{\left[\left(k_{1}-p\right)^{2}+m_{1}^{2}\right]\left[k_{2}^{2}+m_{2}^{2}\right]}\right|_{p^{2}=-m_{3}^{2}},
\end{aligned}
$$

\footnotetext{
${ }^{1}$ There is a typo in Eq. (4.10) of [9]: the coefficient before $\pi \operatorname{Ls}_{4}\left(\frac{\pi}{3}\right)$ should be $\frac{161}{54}$ instead of $\frac{161}{154}$. We are grateful to Y. Schröder and A. Vuorinen for correspondence.
} 


$$
A_{0}(m)=\int \frac{d^{n} k_{1}}{k_{1}^{2}+m^{2}} \equiv \frac{4 m^{n-2}}{(n-2)(n-4)}
$$

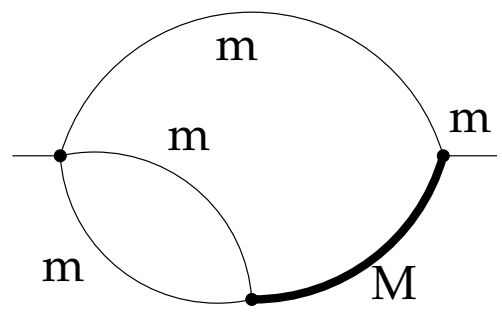

$\mathrm{V}_{\mathrm{mmmM}}$

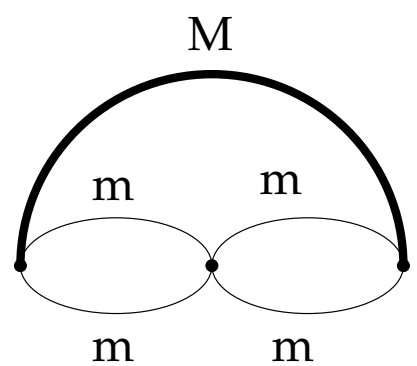

$\mathrm{D}_{\mathrm{mmmmM}}$

Figure 1:

\section{$2 V_{m m m M}$}

This integral enters in two-loop relation between pole and $\overline{M S}$ masses of heavy particles like t-quark or Higgs boson within SM $[23,24]$. The integrals with an arbitrary set of indices,

$$
V_{m m m M}(\alpha, \beta, \sigma, \lambda)=\int \frac{d^{n} k_{1} d^{n} k_{2}}{\left(\left(p-k_{1}\right)^{2}+m^{2}\right)^{\sigma}\left(\left(k_{1}-k_{2}\right)^{2}+m^{2}\right)^{\alpha}\left(k_{2}^{2}+m^{2}\right)^{\beta}\left(k_{1}^{2}+M^{2}\right)^{\lambda}}
$$

where the external moment belongs to the mass shell, $p^{2}=-m^{2}$, can be reduced to one master-integral with indices $(1,1,1,1)$. For the calculation of the diagram, we use the differential equation technique [25]

$$
\begin{aligned}
& \frac{d}{d M^{2}} V_{m m m M}(1,1,1,1)=V_{m m m M}(1,1,1,1)\left[\left(\frac{n}{2}-2\right) \frac{1}{M^{2}}+\frac{(n-3)}{M^{2}-4 m^{2}}\right] \\
& -\left[V_{M m m}+2 A_{0}(m) B_{0}(M, m, m)\right] \frac{1}{4 m^{2}}\left(\frac{n}{2}-1\right)\left(\frac{1}{M^{2}}-\frac{1}{M^{2}-4 m^{2}}\right) \\
& +J_{m m m} \frac{1}{m^{2}}\left(\frac{3 n}{8}-1\right)\left(\frac{1}{M^{2}}-\frac{1}{M^{2}-4 m^{2}}\right) .
\end{aligned}
$$

The analytical solution of this equation up to part linear in $\varepsilon$ was presented in [24]. Equation (2.3) has a very simple form in the framework of the geometrical approach [26]. Let us introduce a new variable

$$
\cos \theta=\frac{M}{2 m}, \quad M \leq 2 m
$$


Taking into account all order $\varepsilon$-expansion of the one-loop propagator and two-loop bubble integrals $[27,8]$ we rewrite the original differential equation (2.3)

$$
\begin{aligned}
& \frac{1}{\sin \theta \cos \theta} \frac{d}{d \theta} V_{m m m M}-2\left(\frac{\varepsilon}{\cos ^{2} \theta}+\frac{1-2 \varepsilon}{\sin ^{2} \theta}\right) V_{m m m M} \\
& -\frac{1}{2 \cos ^{2} \theta \sin ^{2} \theta(1-2 \varepsilon)}\left\{-\frac{1}{\varepsilon^{2}}\left[3-6 \cos ^{2} \theta+2\left(4 \cos ^{2} \theta\right)^{1-\varepsilon}\right]\right. \\
& \left.+\frac{2 \theta}{\varepsilon}(4 \cos \theta \sin \theta)^{1-2 \varepsilon}+(4 \cos \theta \sin \theta)^{1-2 \varepsilon} \sum_{j=0}^{\infty} \frac{(2 \varepsilon)^{j}}{(j+1) !}\left[4 \operatorname{Ls}_{j+2}(2 \theta)-3 \operatorname{Ls}_{j+2}(4 \theta)\right]\right\} \\
& +\frac{J_{m m m}}{m^{2}} \frac{2-3 \varepsilon}{2 \sin ^{2} \theta \cos ^{2} \theta}=0 .
\end{aligned}
$$

For a modified function, $\tilde{V}(\theta)$ defined as

$$
V_{m m m M}=(\sin \theta)^{2-4 \varepsilon}(\cos \theta)^{-2 \varepsilon}\left(m^{2}\right)^{-2 \varepsilon} \tilde{V}(\theta),
$$

the solution has the following form:

$$
\begin{aligned}
& 2(1-2 \varepsilon)^{2} \tilde{V}(\theta)=-(2-3 \varepsilon)(1-2 \varepsilon) \frac{J_{m m m}}{\left(m^{2}\right)^{1-2 \varepsilon}}\left[(1-3 \varepsilon) \operatorname{Isc}_{4,2}(\theta)-\frac{(\sin \theta)^{4 \varepsilon}(\cos \theta)^{2 \varepsilon}}{2 \sin ^{2} \theta}\right] \\
& -\frac{1}{\varepsilon^{2}}\left[3(1-\varepsilon) \operatorname{Isc}_{4,2}(\theta)+\frac{3}{2} \frac{(\sin \theta)^{4 \varepsilon}(\cos \theta)^{2 \varepsilon}}{\sin ^{2} \theta}-4^{1-\varepsilon} \frac{(\sin \theta)^{4 \varepsilon}}{\sin ^{2} \theta}\right] \\
& +4^{1-2 \varepsilon} \sum_{j=0}^{\infty} \frac{(2 \varepsilon)^{j-1}}{j !}\left\{-\frac{\cos \theta}{(\sin \theta)^{1-2 \varepsilon}}\left[4 \operatorname{Ls}_{j+1}(2 \theta)-3 \operatorname{Ls}_{j+1}(4 \theta)\right]\right. \\
& -2^{-2 \varepsilon} \sum_{k=0}^{\infty} \frac{(2 \varepsilon)^{k}}{k !}\left[8 \frac{\ln ^{k+j+1}(2 \sin \theta)}{k+j+1}+3 \varepsilon \operatorname{Ls}_{k+1}(2 \theta) \operatorname{Ls}_{j+1}(4 \theta)\right] \\
& -2^{2-2 \varepsilon} \varepsilon \sum_{k=0}^{\infty} \frac{(2 \varepsilon)^{k}}{k !} \int^{\theta} d \phi\left[2 \ln { }^{k}(2 \sin \phi) \operatorname{Ls}_{j+1}(2 \phi)+3 \operatorname{Ls}_{k+1}(2 \phi)[\ln (2 \sin \phi)+\ln (2 \cos \phi)]^{j}\right] \\
& \left.+3 \cdot 4^{1-\varepsilon} \sum_{k=0}^{\infty} \frac{(2 \varepsilon)^{k}}{k !} \int^{\theta} d \phi \frac{\cos \phi}{\sin \phi} \ln ^{k}(2 \sin \phi)[\ln (2 \sin \phi)+\ln (2 \cos \phi)]^{j}\right\},
\end{aligned}
$$

where $\operatorname{Ls}_{j}(\theta)$ is a log-sine function [21] and we have introduced a new function $\operatorname{Isc}_{a, b}(\theta)$

$$
\operatorname{Isc}_{a, b}(\theta)=\int^{\theta} d \phi \frac{(\sin \phi)^{a \varepsilon}(\cos \phi)^{b \varepsilon}}{\sin \phi \cos \phi}
$$

where $a, b$ are integer numbers. The $\varepsilon$-expansion of this function is

$$
\begin{aligned}
& \operatorname{Isc}_{a, b}(\theta)=\ln (\tan \theta)+\frac{1}{2} \sum_{i=1}^{\infty} \frac{\left(\frac{\varepsilon}{2}\right)^{i}}{(i+1) !}\left(a^{i} \ln ^{i+1}\left(\sin ^{2} \theta\right)-b^{i} \ln ^{i+1}\left(\cos ^{2} \theta\right)\right) \\
& -\frac{1}{2} \sum_{i=1}^{\infty}\left(-\frac{\varepsilon}{2}\right)^{i}\left(a^{i} \mathrm{~S}_{1, i}\left(\cos ^{2} \theta\right)-b^{i} \mathrm{~S}_{1, i}\left(\sin ^{2} \theta\right)\right)
\end{aligned}
$$




$$
\begin{aligned}
+\frac{1}{2} \sum_{i, j=1}^{\infty} a^{i} b^{j}\left(\frac{-\varepsilon}{2}\right)^{(i+j)} & {\left[\sum_{r=0}^{i} \frac{(-1)^{r} \ln ^{r}\left(\sin ^{2} \theta\right)}{r !} \mathrm{S}_{i+1-r, j}\left(\sin ^{2} \theta\right)\right.} \\
& \left.-\sum_{r=0}^{j} \frac{(-1)^{r} \ln ^{r}\left(\cos ^{2} \theta\right)}{r !} \mathrm{S}_{j+1-r, i}\left(\cos ^{2} \theta\right)\right]
\end{aligned}
$$

where $\mathrm{S}_{a, b}(\theta)$ is the generalized (Nielsen) polylogarithms [10]. The last integral in Eq. (2.7) can be explicitly integrated

$$
\begin{aligned}
& \int^{\theta} d \theta \frac{\cos \theta}{\sin \theta} \ln ^{a}(\sin \theta)=\frac{1}{1+a} \ln ^{a+1} \sin \theta \\
& \int^{\theta} d \theta \frac{\cos \theta}{\sin \theta} \ln ^{a}(\sin \theta) \ln ^{b}(\cos \theta)=\frac{b !(-1)^{b}}{2^{1+a+b}} \sum_{i=0}^{a} \frac{a !(-1)^{i}}{(a-i) !}(2 \ln (\sin \theta))^{a-i} \mathrm{~S}_{i+1, b}\left(\sin ^{2} \theta\right)
\end{aligned}
$$

The solution for the original integral can be represented as

$$
V_{m m m M}=(\sin \theta)^{2-4 \varepsilon}(\cos \theta)^{-2 \varepsilon}\left(m^{2}\right)^{-2 \varepsilon}\left\{\tilde{V}(\theta)+\sum_{j=-1}^{\infty} \varepsilon^{j} v_{j}\right\},
$$

where the coefficients $v_{j}$ should be defined from the boundary condition. We choose a particular value of the diagram at $M=0$ which corresponds to $\theta=\pi / 2$. In this case the diagram reduces to $J_{m m m}$ and the product of one-loop vacuum diagrams

$$
\left.V_{m m m M}\right|_{M=0}=\frac{3 n-8}{4(4-n)} \frac{J_{m m m}}{m^{2}}-\frac{3(n-2)^{2}}{8(n-4)(n-3)}\left[\frac{A_{0}\left(m^{2}\right)}{m^{2}}\right]^{2} .
$$

Using the results of $[11,28]$ we get the following values for the first several coefficients:

$$
\begin{aligned}
v_{-1}= & 12 \ln 2, \quad v_{0}=48 \ln 2+6 \zeta_{2}-36 \ln ^{2} 2, \\
v_{1}= & 144 \ln 2+24 \zeta_{2}-5 \zeta_{3}-12 \zeta_{2} \ln 2-144 \ln ^{2} 2+72 \ln ^{3} 2, \\
v_{2}= & 384 \ln 2+72 \zeta_{2}-20 \zeta_{3}-29 \zeta_{4}-48 \zeta_{2} \ln 2+24 \zeta_{2} \ln ^{2} 2 \\
& -432 \ln ^{2} 2+288 \ln ^{3} 2-106 \ln ^{4} 2+48 \operatorname{Li}_{4}\left(\frac{1}{2}\right), \\
v_{3}= & 960 \ln 2+192 \zeta_{2}-60 \zeta_{3}-116 \zeta_{4}-\frac{633}{2} \zeta_{5}-144 \zeta_{2} \ln 2+96 \zeta_{2} \ln ^{2} 2 \\
& -48 \zeta_{2} \ln ^{3} 2+306 \zeta_{4} \ln 2-1152 \ln ^{2} 2+864 \ln ^{3} 2-424 \ln ^{4} 2+\frac{636}{5} \ln ^{5} 2 \\
& +192 \operatorname{Li}_{4}\left(\frac{1}{2}\right)+288 \operatorname{Li}_{5}\left(\frac{1}{2}\right) .
\end{aligned}
$$

$v_{4}$ can also be calculated by using the results of [28].

From Eq. (2.7) it is evident, that the coefficients of the $\varepsilon$-expansion of the diagram $V_{1112}$ can be parametrized by introducing a new class of functions $\operatorname{LsLsc}_{k, i, j}(\theta)$ defined as

$$
\operatorname{LsLsc}_{k, i, j}(\theta)=\int_{0}^{\theta} d \phi \operatorname{Ls}_{k+1}(\phi) \ln ^{i-1}\left|2 \sin \frac{\phi}{2}\right| \ln ^{j-1}\left|2 \cos \frac{\phi}{2}\right|,
$$

where $k, i, j$ are integer numbers, $k \geq 0$ and $i, j \geq 1$. Some properties of these functions are collected in Appendix B. 
Let us write the $\varepsilon$-expansion of the diagram in following form:

$V_{m m m M}(\theta)=\left(m^{2}\right)^{-2 \varepsilon}\left[\frac{1}{2 \varepsilon^{2}}+\frac{1}{\varepsilon} S(\theta)+F(\theta)+\varepsilon E(\theta)+\varepsilon^{2} N(\theta)+\varepsilon^{3} N_{n}(\theta)+\varepsilon^{4} N_{n n}(\theta)+\mathcal{O}\left(\varepsilon^{5}\right)\right]$.

The functions $S(\theta), F(\theta)$ and $E(\theta)$ were calculated in [24] (see Eq.(3.27)). The analytical results for $N(\theta)$ for arbitrary values of the angle $\theta$ read

$$
\begin{aligned}
& \frac{N(\theta)}{\sin ^{2} \theta}=4 \frac{F(\theta)-E(\theta)}{\sin ^{2} \theta}-4 \theta\left[4 \mathrm{Ls}_{3}(2 \theta)-3 \mathrm{Ls}_{3}(4 \theta)\right] \\
& -\frac{2}{3} \frac{\sin (2 \theta)}{\sin ^{2} \theta} \sum_{j=0}^{3}(-1)^{j}\left[4 \mathrm{Ls}_{4-j}(2 \theta)-3 \mathrm{Ls}_{4-j}(4 \theta)\right] l_{2 \theta}^{j}\left(\begin{array}{l}
3 \\
j
\end{array}\right) \\
& +\frac{1}{\sin ^{2} \theta}\left\{\frac{27}{2}+\frac{4}{3} \cos ^{2} \theta L_{\theta}^{4}+24 \zeta_{2} \ln 2-14 \zeta_{3}-12 \zeta_{2}\right\} \\
& -24 \Phi(2 \theta)+12 \mathrm{Ls}_{4}^{(1)}(2 \theta)-3 \mathrm{Ls}_{4}^{(1)}(4 \theta)+20\left[\mathrm{Ls}_{2}(2 \theta)\right]^{2}-12 \mathrm{Ls}_{2}(2 \theta) \mathrm{Ls}_{2}(4 \theta) \\
& -16 \theta L_{\theta} \mathrm{Ls}_{2}(2 \theta)-32 \theta l_{\theta} \mathrm{Ls}_{2}(2 \theta)+24 \mathrm{Cl}_{3}(\pi-2 \theta)\left[L_{\theta}+2 l_{\theta}\right]-12 \zeta_{2} \mathrm{Li}_{2}\left(\sin ^{2} \theta\right) \\
& -8 \theta^{2} L_{\theta}^{2}-32 \theta^{2} l_{\theta}^{2}-32 \theta^{2} L_{\theta} l_{\theta}+36 \zeta_{3} l_{\theta}-24 \zeta_{3} L_{\theta}+12 \zeta_{2} L_{\theta}^{2}+48 \ln 2 \zeta_{2} L_{\theta} \\
& +42 \zeta_{3} \ln 2-31 \zeta_{4}+2 \ln ^{4} 2-36 \zeta_{2} \ln ^{2} 2+48 \mathrm{Li}_{4}\left(\frac{1}{2}\right),
\end{aligned}
$$

where

$$
L_{\theta}=\ln (2 \cos \theta), \quad l_{\theta}=\ln (2 \sin \theta), \quad l_{m \theta}=\ln (2 \sin m \theta),
$$

and

$$
\Phi(\theta) \equiv \int_{0}^{\theta} d \phi \operatorname{Ls}_{2}(\phi) \ln \left(2 \cos \frac{\phi}{2}\right)=\operatorname{LsLsc}_{1,1,2}(\theta),
$$

is the function defined in Eq. (2.41) of [29]. The results for $N_{n}(\theta)$ and $N_{n n}(\theta)$ are sufficiently lengthy to be published here. It should be mentioned that in the order of $\varepsilon^{3}$ the following combination appears:

$2 \operatorname{LsLsc}_{1,2,2}(2 \theta)+\operatorname{LsLsc}_{0,3,2}(2 \theta)+\operatorname{LsLsc}_{0,3,2}(\pi-2 \theta)+\operatorname{LsLsc}_{1,1,3}(2 \theta)-\operatorname{LsLsc}_{1,1,3}(\pi-2 \theta)$

To write results valid in other regions of the variable $(M>2 m)$, a proper analytical continuation of all expressions should be constructed. For generalized log-sine functions it is described in detail in $[9,31]$. In terms of the variable

$$
y \equiv e^{\mathrm{i} \sigma 2 \theta}, \quad \ln (-y-\mathrm{i} \sigma 0)=\ln y-\mathrm{i} \sigma \pi,
$$

the analytical continuation of all generalized log-sine can be expressed in terms of Nielsen polylogarithms, whereas for the function $\Phi(\theta)$ the result is expressible in terms of harmonic polylogarithms (see Eq.(3.4) in [29]) introduced by Remiddi and Vermaseren [32]. In terms of conformal variable (2.18), the analytical continuation of the LsLsc functions can be written 
in terms of harmonic polylogarithms (see the discussion in $[9,29,33]$ ). In particular, an analytical continuation of the $\operatorname{LsLsc}_{1,1,3}(\theta)$-function produces integral $\int H_{-1,0,0,1}(z) \frac{d z}{1+z}$. The result is relatively lengthy to be published here. The analytically continued results of the order $\varepsilon^{2}$ checked by heavy mass expansion [34] with the help of the packages described in [35].

For a particular case $M=m$ the integral $V_{m m m M}$ converts into the master-integral $V_{1111}$ from the package ONSHELL2 $[3,30]$. Its $\varepsilon$-expansion is (we present only new coefficients of the expansion)

$$
\begin{aligned}
& N\left(\frac{\pi}{3}\right) \equiv V_{1111}\left[\varepsilon^{2}\right]=\frac{211}{2}-32 \frac{\pi}{\sqrt{3}}+12 \frac{\pi}{\sqrt{3}} \ln 3-2 \frac{\pi}{\sqrt{3}} \ln ^{2} 3+\frac{1}{6} \frac{\pi}{\sqrt{3}} \ln ^{3} 3 \\
& -34 \zeta_{2}+16 \zeta_{2} \ln 3-4 \zeta_{2} \ln ^{2} 3-32 \zeta_{3}+\frac{39}{2} \zeta_{3} \ln 3-18 \zeta_{2} \frac{\pi}{\sqrt{3}}+\frac{9}{2} \zeta_{2} \frac{\pi}{\sqrt{3}} \ln 3+9 \zeta_{3} \frac{\pi}{\sqrt{3}} \\
& -84 \frac{\operatorname{Ls}_{2}\left(\frac{\pi}{3}\right)}{\sqrt{3}}+28 \frac{\mathrm{Ls}_{2}\left(\frac{\pi}{3}\right)}{\sqrt{3}} \ln 3+\frac{16}{3} \pi \mathrm{Ls}_{2}\left(\frac{\pi}{3}\right)-42 \frac{\mathrm{Ls}_{3}\left(\frac{2 \pi}{3}\right)}{\sqrt{3}} \\
& -\frac{7}{2} \frac{\mathrm{Ls}_{2}\left(\frac{\pi}{3}\right)}{\sqrt{3}} \ln ^{2} 3-\frac{8}{3} \pi \mathrm{Ls}_{2}\left(\frac{\pi}{3}\right) \ln 3+\frac{21}{2} \frac{\mathrm{Ls}_{3}\left(\frac{2 \pi}{3}\right)}{\sqrt{3}} \ln 3-7 \pi \mathrm{Ls}_{3}\left(\frac{2 \pi}{3}\right) \\
& -\frac{219}{4} \zeta_{4}-7 \frac{\mathrm{Ls}_{4}\left(\frac{2 \pi}{3}\right)}{\sqrt{3}}+\frac{27}{2} \mathrm{Ls}_{4}^{(1)}\left(\frac{2 \pi}{3}\right)+\frac{14}{3}\left[\mathrm{Ls}_{2}\left(\frac{\pi}{3}\right)\right]^{2} \\
& +24 \zeta_{2} \ln 2+18 \zeta_{2} \ln ^{2} 2-18 \zeta_{2} \ln 2 \ln 3+9 \zeta_{2} \mathrm{Li}_{2}\left(\frac{1}{4}\right),
\end{aligned}
$$

and

$$
\begin{aligned}
& N_{n}\left(\frac{\pi}{3}\right) \equiv V_{1111}\left[\varepsilon^{3}\right]=\frac{665}{2}-80 \frac{\pi}{\sqrt{3}}+32 \frac{\pi}{\sqrt{3}} \ln 3-6 \frac{\pi}{\sqrt{3}} \ln ^{2} 3+\frac{2}{3} \frac{\pi}{\sqrt{3}} \ln ^{3} 3-\frac{1}{24} \frac{\pi}{\sqrt{3}} \ln ^{4} 3 \\
& -54 \zeta_{2} \frac{\pi}{\sqrt{3}}+18 \zeta_{2} \frac{\pi}{\sqrt{3}} \ln 3-\frac{9}{4} \zeta_{2} \frac{\pi}{\sqrt{3}} \ln ^{2} 3-148 \zeta_{2}-152 \zeta_{3}-157 \zeta_{4}+\frac{1081}{24} \zeta_{2} \zeta_{3}+\frac{3593}{12} \zeta_{5} \\
& +48 \zeta_{2} \ln 3-16 \zeta_{2} \ln ^{2} 3+\frac{8}{3} \zeta_{2} \ln ^{3} 3+36 \zeta_{3} \frac{\pi}{\sqrt{3}}-9 \zeta_{3} \frac{\pi}{\sqrt{3}} \ln 3+78 \zeta_{3} \ln 3-\frac{39}{2} \zeta_{3} \ln ^{2} 3 \\
& +63 \zeta_{4} \ln 3-\frac{171}{8} \zeta_{4} \frac{\pi}{\sqrt{3}}-224 \frac{\operatorname{Ls}_{2}\left(\frac{\pi}{3}\right)}{\sqrt{3}}+84 \frac{\operatorname{Ls}_{2}\left(\frac{\pi}{3}\right)}{\sqrt{3}} \ln 3-14 \frac{\operatorname{Ls}_{2}\left(\frac{\pi}{3}\right)}{\sqrt{3}} \ln ^{2} 3+\frac{7}{6} \frac{\operatorname{Ls}_{2}\left(\frac{\pi}{3}\right)}{\sqrt{3}} \ln ^{3} 3 \\
& +16 \pi \operatorname{Ls}_{2}\left(\frac{\pi}{3}\right)-\frac{32}{3} \pi \operatorname{Ls}_{2}\left(\frac{\pi}{3}\right) \ln 3+\frac{8}{3} \pi \operatorname{Ls}_{2}\left(\frac{\pi}{3}\right) \ln ^{2} 3+6 \pi \zeta_{2} \operatorname{Ls}_{2}\left(\frac{\pi}{3}\right) \\
& -126 \frac{\operatorname{Ls}_{3}\left(\frac{2 \pi}{3}\right)}{\sqrt{3}}+42 \frac{\operatorname{Ls}_{3}\left(\frac{2 \pi}{3}\right)}{\sqrt{3}} \ln 3-\frac{21}{4} \frac{\operatorname{Ls}_{3}\left(\frac{2 \pi}{3}\right)}{\sqrt{3}} \ln ^{2} 3-14 \pi \operatorname{Ls}_{3}\left(\frac{2 \pi}{3}\right)[2-\ln 3] \\
& +\frac{23}{18} \pi \operatorname{Ls}_{4}\left(\frac{\pi}{3}\right)-\frac{23}{3} \pi \operatorname{Ls}_{4}\left(\frac{2 \pi}{3}\right)-7 \frac{\operatorname{Ls}_{4}\left(\frac{2 \pi}{3}\right)}{\sqrt{3}}[4-\ln 3]+27 \operatorname{Ls}_{4}^{(1)}\left(\frac{2 \pi}{3}\right)[2-\ln 3] \\
& -\frac{7}{2} \frac{\operatorname{Ls}_{5}\left(\frac{2 \pi}{3}\right)}{\sqrt{3}}+\frac{28}{3}\left[\operatorname{Ls}_{2}\left(\frac{\pi}{3}\right)\right]^{2}[2-\ln 3]-4 \operatorname{Ls}_{2}\left(\frac{\pi}{3}\right) \operatorname{Ls}_{3}\left(\frac{2 \pi}{3}\right)-\frac{55}{6} \pi \zeta_{2} \operatorname{Ls}_{2}\left(\frac{\pi}{3}\right) \\
& +9 \operatorname{Ls}_{5}^{(1)}\left(\frac{2 \pi}{3}\right)-\frac{9}{8} \chi_{5}+36 \operatorname{LsLsc}_{1,1,3}\left(\frac{\pi}{3}\right)+168 \zeta_{2} \ln 2 \\
& -4 \ln ^{4} 2+24 \zeta_{2} \ln ^{2} 2-96 \mathrm{Li}_{4}\left(\frac{1}{2}\right)-\frac{48}{5} \ln ^{5} 2-63 \zeta_{3} \ln ^{2} 2-288\left[\operatorname{Li}_{4}\left(\frac{1}{2}\right) \ln 2+\mathrm{Li}_{5}\left(\frac{1}{2}\right)\right] \\
& +\mathrm{Li}_{2}\left(\frac{1}{4}\right)\left[36 \zeta_{2}+\frac{63}{2} \zeta_{3}-18 \zeta_{2} \ln 2-18 \zeta_{2} \ln 3\right]-9 \zeta_{2} \mathrm{Li}_{3}\left(\frac{1}{4}\right)-18 \zeta_{2} \mathrm{~S}_{1,2}\left(\frac{1}{4}\right) \\
& +72 \mathrm{Li}_{4}\left(\frac{1}{2}\right) \ln 3+3 \ln 3 \ln ^{4} 2-72 \zeta_{2} \ln 2 \ln 3+18 \zeta_{2} \ln 2 \ln ^{2} 3 .
\end{aligned}
$$

Numerical values of the coefficients, $V_{1111}\left[\varepsilon^{2}\right]$ and $V_{1111}\left[\varepsilon^{3}\right]$ are in full agreement with the proper results of [17]. 


\section{$3 \quad D_{m m m m M}$}

The differential equation for this integral is

$$
\begin{aligned}
& -M^{2} \frac{d}{d M^{2}} D_{m m m m M}=\left(5+4(3-n) \frac{m^{2}}{M^{2}-4 m^{2}}-\frac{3}{2} n\right) D_{m m m m M} \\
& +B_{N}\left(\frac{3}{2} n-4\right) \frac{1}{M^{2}-4 m^{2}}+V_{M m m} A_{0}(m) \frac{4-2 n}{M^{2}-4 m^{2}},
\end{aligned}
$$

where $B_{N} \equiv B_{N}(0,0,1,1,1,1)$ was defined by Broadhurst in $[11,12]$. Using again the angle variable defined in (2.4), we rewrite equation (3.21) in the following form:

$$
\begin{aligned}
& \frac{\cos \theta}{2 \sin \theta} \frac{d}{d \theta} D_{m m m m M}-\left(-1+3 \varepsilon+\frac{1-2 \varepsilon}{\sin ^{2} \theta}\right) D_{m m m m M} \\
& =-\frac{B_{N}}{m^{2}} \frac{(2-3 \varepsilon)}{4} \frac{1}{\sin ^{2} \theta}-\frac{\left(m^{2}\right)^{1-3 \varepsilon}}{\sin ^{2} \theta} \frac{1}{\varepsilon(1-\varepsilon)(1-2 \varepsilon)}\left\{-\frac{1}{\varepsilon^{2}}\left[1-2 \cos ^{2} \theta+\left(4 \cos ^{2} \theta\right)^{1-\varepsilon}\right]\right. \\
& \left.+(4 \cos \theta \sin \theta)^{1-2 \varepsilon} \sum_{j=0}^{\infty} \frac{(2 \varepsilon)^{j}}{(j+1) !}\left[2 \operatorname{Ls}_{j+2}(2 \theta)-\mathrm{Ls}_{j+2}(4 \theta)\right]\right\}
\end{aligned}
$$

For auxiliary function $\tilde{D}(\theta)$ defined as

$$
D_{m m m m M}=(\sin \theta)^{2-4 \varepsilon}(\cos \theta)^{-2 \varepsilon}\left(m^{2}\right)^{1-3 \varepsilon} \tilde{D}(\theta)
$$

the solution is

$$
\begin{aligned}
& (1-\varepsilon)(1-2 \varepsilon)^{2} \tilde{D}(\theta)= \\
& -\frac{(1-\varepsilon)(2-3 \varepsilon)(1-2 \varepsilon)}{2} \frac{B_{N}}{\left(m^{2}\right)^{2-3 \varepsilon}}\left[(1-3 \varepsilon) \operatorname{Isc}_{4,2}(\theta)-\frac{(\sin \theta)^{4 \varepsilon}(\cos \theta)^{2 \varepsilon}}{2 \sin ^{2} \theta}\right] \\
& +\frac{2}{\varepsilon^{3}}\left[(1-\varepsilon) \operatorname{Isc}_{4,2}(\theta)+\frac{(\sin \theta)^{4 \varepsilon}(\cos \theta)^{2 \varepsilon}}{2 \sin ^{2} \theta}-2^{1-2 \varepsilon} \frac{(\sin \theta)^{4 \varepsilon}}{\sin ^{2} \theta}\right] \\
& -2^{3-4 \varepsilon} \frac{1}{\varepsilon} \sum_{j=0}^{\infty} \frac{(2 \varepsilon)^{j}}{(j+1) !}\left\{-\frac{\cos \theta}{(\sin \theta)^{1-2 \varepsilon}}\left[2 \operatorname{Ls}_{j+2}(2 \theta)-\operatorname{Ls}_{j+2}(4 \theta)\right]\right. \\
& -2^{-2 \varepsilon} \sum_{k=0}^{\infty} \frac{(2 \varepsilon)^{k}}{k !}\left[4 \frac{\ln { }^{k+j+2}(2 \sin \theta)}{k+j+2}+\varepsilon \operatorname{Ls}_{k+1}(2 \theta) \operatorname{Ls}_{j+2}(4 \theta)\right] \\
& -2^{2-2 \varepsilon} \varepsilon \sum_{k=0}^{\infty} \frac{(2 \varepsilon)^{k}}{k !} \int^{\theta} d \phi\left[\operatorname{Ls} k+1(2 \phi)[\ln (2 \sin \phi)+\ln (2 \cos \phi)]^{j+1}+\ln ^{k}(2 \sin \phi) \operatorname{Ls}_{j+2}(2 \phi)\right] \\
& \left.+2^{2-2 \varepsilon} \sum_{k=0}^{\infty} \frac{(2 \varepsilon)^{k}}{k !} \int^{\theta} d \phi \frac{\cos \phi}{\sin \phi} \ln ^{k}(2 \sin \phi)[\ln (2 \sin \phi)+\ln (2 \cos \phi)]^{j+1}\right\}
\end{aligned}
$$

where the last integral can be explicitly integrated with the help of Eq. (2.10) and $\operatorname{Isc}_{a, b}(\theta)$ is defined in (2.9). The result for the diagram $D_{m m m m M}$ is

$$
D_{m m m m M}=(\sin \theta)^{2-4 \varepsilon}(\cos \theta)^{-2 \varepsilon}\left(m^{2}\right)^{1-3 \varepsilon}\left\{\tilde{D}(\theta)+\sum_{j=-3}^{\infty} \varepsilon^{j} d_{j}\right\},
$$


where the coefficients $d_{j}$ are defined from the value of the original diagrams at $M=0$, which corresponds to $\theta=\pi / 2$ :

$$
\left.m^{2} D_{m m m m M}\right|_{M=0}=V_{0 m m} A_{0}(m) \frac{n-2}{n-4}-B_{N} \frac{3 n-8}{4(n-4)} .
$$

The explicit values of the first several coefficients are

$$
\begin{aligned}
d_{-3}= & \frac{4}{3}, \quad d_{-2}=\frac{20}{3}-8 \ln 2, \quad d_{-1}=\frac{68}{3}-\frac{16}{3} \zeta_{2}-40 \ln 2-8 \ln ^{2} 2 \\
d_{0}= & \frac{196}{3}+\frac{20}{3} \zeta_{3}-\frac{80}{3} \zeta_{2}-136 \ln 2-40 \ln ^{2} 2+\frac{176}{3} \ln ^{3} 2 \\
d_{1}= & -\frac{400}{3} \ln ^{4} 2+\frac{880}{3} \ln ^{3} 2+16 \zeta_{2} \ln ^{2} 2-136 \ln ^{2} 2-392 \ln 2 \\
& -\frac{272}{3} \zeta_{2}+\frac{100}{3} \zeta_{3}+\frac{116}{3} \zeta_{4}-64 \operatorname{Li}_{4}\left(\frac{1}{2}\right)+172 \\
d_{2}= & 428+\frac{2912}{15} \ln ^{5} 2-\frac{2000}{3} \ln ^{4} 2+\frac{2992}{3} \ln ^{3} 2-392 \ln ^{2} 2-1032 \ln 2 \\
& -32 \zeta_{2} \ln ^{3} 2+80 \zeta_{2} \ln ^{2} 2-408 \zeta_{4} \ln 2-\frac{784}{3} \zeta_{2}+\frac{340}{3} \zeta_{3}+\frac{580}{3} \zeta_{4} \\
& +\frac{16}{3} \zeta_{2} \zeta_{3}+422 \zeta_{5}-320 \operatorname{Li}_{4}\left(\frac{1}{2}\right)-384 \operatorname{Li}_{5}\left(\frac{1}{2}\right),
\end{aligned}
$$

The next coefficient $d_{3}$ contains the $U_{5,1}$ constant [12] which could be rewritten in terms of the generalized log-sine function of the argument $\pi / 2$ [9]. Collecting all the results we get the first several coefficients of the $\varepsilon$-expansion of the diagram

$$
\begin{aligned}
\left(m^{2}\right)^{-1+3 \varepsilon}(1-\varepsilon)(1-2 \varepsilon)^{2} D_{m m m m M}(\theta)=-\frac{2}{3 \varepsilon^{3}}\left\{1+2 \cos ^{2} \theta\right\}-\frac{2}{3 \varepsilon^{2}}\left\{1-12 \cos ^{2} \theta L_{\theta}\right\} \\
-\frac{2}{\varepsilon}\left\{1+4 \cos ^{2} \theta L_{\theta}^{2}-2 \sin (2 \theta)\left[2 \mathrm{Ls}_{2}(2 \theta)-\mathrm{Ls}_{2}(4 \theta)\right]\right\} \\
\quad-6+\frac{16}{3} \cos ^{2} \theta L_{\theta}^{3}-4 \sin ^{2} \theta\left[4 \mathrm{Cl}_{3}(2 \theta)-\mathrm{Cl}_{3}(4 \theta)-\frac{2}{3} \zeta_{3}\right] \\
\quad+4 \sin (2 \theta)\left[2 \mathrm{Ls}_{3}(2 \theta)-\mathrm{Ls}_{3}(4 \theta)\right]-8 \sin (2 \theta) l_{2 \theta}\left[2 \mathrm{Ls}_{2}(2 \theta)-\mathrm{Ls}_{2}(4 \theta)\right] \\
+\varepsilon\left\{-18+\frac{56}{3} \zeta_{3}+8 \sin ^{2} \theta\left[2 l_{\theta}+L_{\theta}\right]\left[4 \mathrm{Cl}_{3}(2 \theta)-\mathrm{Cl}_{3}(4 \theta)\right]\right. \\
\quad+4 \sin ^{2} \theta\left[8 F(2 \theta)-4 \mathrm{Ls}_{4}^{(1)}(2 \theta)+\mathrm{Ls}_{4}^{(1)}(4 \theta)\right] \\
\quad+16 \sin ^{2} \theta\left[\theta\left[2 \mathrm{Ls}_{3}(2 \theta)-\mathrm{Ls}_{3}(4 \theta)\right]-\mathrm{Ls}_{2}(2 \theta)\left[2 \mathrm{Ls}_{2}(2 \theta)-\mathrm{Ls}_{2}(4 \theta)\right]+\zeta_{3}\left(2 L_{\theta}-3 l_{\theta}\right)\right] \\
\quad+4 \sin ^{2} \theta\left[17 \zeta_{4}-16 \mathrm{Li}_{4}\left(\frac{1}{2}\right)+4 \zeta_{2} \ln ^{2} 2-14 \zeta_{3} \ln 2-\frac{2}{3} \ln ^{4} 2\right]-\frac{8}{3} \cos ^{2} \theta L_{\theta}^{4} \\
\quad+8 \sin (2 \theta) l_{2 \theta}^{2}\left[2 \mathrm{Ls}_{2}(2 \theta)-\mathrm{Ls}_{2}(4 \theta)\right]-8 \sin (2 \theta) l_{2 \theta}\left[2 \mathrm{Ls}_{3}(2 \theta)-\mathrm{Ls}_{3}(4 \theta)\right] \\
\left.\quad+\frac{8}{3} \sin (2 \theta)\left[2 \mathrm{Ls}_{4}(2 \theta)-\mathrm{Ls}_{4}(4 \theta)\right]\right\}+\mathcal{O}\left(\varepsilon^{2}\right),
\end{aligned}
$$

where $l_{m \theta}$ and $L_{\theta}$ are defined in (2.16). The analytical results for the next two coefficients are expressible in terms of the LsLsc-functions. In the order $\varepsilon^{2}$ the previous combination (2.17) of LsLsc functions is included. The divergent parts of this integral were calculated previously in [36]. In the regions of the variable $(M>2 m)$ the proper analytical continuation of all 
expressions can be constructed. It completly coincides with the previous case $V_{m m m M}$. The result is relatively lengthy and therefore will not be presented here.

For the case of equal masses $M=m$ (the master-integral $D_{5}$ ) we get

$$
\begin{aligned}
& \left(m^{2}\right)^{-1+3 \varepsilon}(1-\varepsilon)(1-2 \varepsilon)^{2} D_{5}=-\frac{1}{\varepsilon^{3}}-\frac{2}{3 \varepsilon^{2}}-\frac{2}{\varepsilon}\left\{1-6 \frac{\mathrm{Ls}_{2}\left(\frac{\pi}{3}\right)}{\sqrt{3}}\right\} \\
& -6+6 \zeta_{2} \frac{\pi}{\sqrt{3}}+6 \zeta_{3}+18 \frac{\operatorname{Ls}_{3}\left(\frac{2 \pi}{3}\right)}{\sqrt{3}}-12 \frac{\operatorname{Ls}_{2}\left(\frac{\pi}{3}\right)}{\sqrt{3}} \ln 3 \\
& -\varepsilon\left\{18+12 \zeta_{3} \frac{\pi}{\sqrt{3}}+6 \zeta_{2} \frac{\pi}{\sqrt{3}} \ln 3-\frac{56}{3} \zeta_{3}+26 \zeta_{3} \ln 3-63 \zeta_{4}-6 \frac{\mathrm{Ls}_{2}\left(\frac{\pi}{3}\right)}{\sqrt{3}} \ln ^{2} 3\right. \\
& \left.+18 \frac{\operatorname{Ls}_{3}\left(\frac{2 \pi}{3}\right)}{\sqrt{3}} \ln 3-12 \pi \operatorname{Ls}_{3}\left(\frac{2 \pi}{3}\right)+18 \operatorname{Ls}_{4}^{(1)}\left(\frac{2 \pi}{3}\right)+8\left[\operatorname{Ls}_{2}\left(\frac{\pi}{3}\right)\right]^{2}-12 \frac{\operatorname{Ls}_{4}\left(\frac{2 \pi}{3}\right)}{\sqrt{3}}\right\} \\
& +\varepsilon^{2}\left\{-54+3 \zeta_{2} \frac{\pi}{\sqrt{3}} \ln ^{2} 3+12 \zeta_{3} \frac{\pi}{\sqrt{3}} \ln 3+56 \zeta_{3}-136 \zeta_{4}-\frac{793}{18} \zeta_{2} \zeta_{3}-\frac{3593}{9} \zeta_{5}\right. \\
& +26 \zeta_{3} \ln ^{2} 3+\frac{57}{2} \zeta_{4} \frac{\pi}{\sqrt{3}}-24 \zeta_{4} \ln 3-2 \frac{\mathrm{Ls}_{2}\left(\frac{\pi}{3}\right)}{\sqrt{3}} \ln ^{3} 3+9 \frac{\mathrm{Ls}_{3}\left(\frac{2 \pi}{3}\right)}{\sqrt{3}} \ln ^{2} 3-12 \frac{\mathrm{Ls}_{4}\left(\frac{2 \pi}{3}\right)}{\sqrt{3}} \ln 3 \\
& +16\left[\operatorname{Ls}_{2}\left(\frac{\pi}{3}\right)\right]^{2} \ln 3+6 \frac{\operatorname{Ls}_{5}\left(\frac{2 \pi}{3}\right)}{\sqrt{3}}+\frac{38}{9} \pi \zeta_{2} \operatorname{Ls}_{2}\left(\frac{\pi}{3}\right)-12 \operatorname{Ls}_{5}^{(1)}\left(\frac{2 \pi}{3}\right)+\frac{3}{2} \chi_{5} \\
& -24 \pi \operatorname{Ls}_{3}\left(\frac{2 \pi}{3}\right) \ln 3-\frac{46}{27} \pi \operatorname{Ls}_{4}\left(\frac{2 \pi}{3}\right)+12 \pi \operatorname{Ls}_{4}\left(\frac{\pi}{3}\right)+36 \operatorname{Ls}_{4}^{(1)}\left(\frac{2 \pi}{3}\right) \ln 3-48 \operatorname{LsLsc}_{1,1,3}\left(\frac{\pi}{3}\right) \\
& -32 \zeta_{2} \ln ^{2} 2+\frac{16}{3} \ln ^{4} 2+128 \mathrm{Li}_{4}\left(\frac{1}{2}\right) \\
& -64 \zeta_{2} \ln ^{3} 2+84 \zeta_{3} \ln ^{2} 2+\frac{64}{5} \ln ^{5} 2+384\left[\ln 2 \mathrm{Li}_{4}\left(\frac{1}{2}\right) \ln 2+\mathrm{Li}_{5}\left(\frac{1}{2}\right)\right] \\
& \left.+24 \zeta_{2} \ln ^{2} 2 \ln 3-42 \zeta_{3} \operatorname{Li}_{2}\left(\frac{1}{4}\right)-96 \mathrm{Li}_{4}\left(\frac{1}{2}\right) \ln 3-4 \ln ^{4} 2 \ln 3\right\} \text {. }
\end{aligned}
$$

As a non-trivial check of these results we established full agreement between the numerical values for the coefficients of $\varepsilon$-expansion of the $D_{5}$-integral with the proper Laporta results $[17]$.

\section{Conclusion}

In this paper, the higher order $\varepsilon$-expansion of the diagrams shown in Fig. (1) have been constructed $(2.7)$, (3.23). The coefficients of the $\varepsilon$-expansion are parametrized in terms of generalized log-sin functions and LsLsc-functions described in Appendix B. At $M=m$ these integrals enter in FORM [38] based packages for calculation of two-loop on-shell self-energy diagrams [3] $\left(V_{1111}\right)$ and three-loop vacuum integrals $[4,5]\left(D_{5}\right)$. The numerical values of the calculated coefficients $(2.19),(2.20)$ and (3.28) coincide with the results presented in [17].

We shown that the basis of transcendental numbers for single scale diagrams with twoand three-massive cuts contains new elements in addition to the odd/even basis of weight 5 constructed in $[14,9]$. Some of these elements are the product of the lowest weight elements 
of the "sixth root of unity" basis:

$$
\begin{aligned}
& \zeta_{2} \times\left\{\ln 2 \ln 3, \ln 2 \ln ^{2} 3, \operatorname{Li}_{3}\left(\frac{1}{4}\right), \mathrm{S}_{1,2}\left(\frac{1}{4}\right)\right\}, \quad \operatorname{Li}_{2}\left(\frac{1}{4}\right) \times\left\{\zeta_{2}, \zeta_{3}, \zeta_{2} \ln 2, \zeta_{2} \ln 3\right\}, \\
& \mathrm{Li}_{4}\left(\frac{1}{2}\right) \times\{\ln 2, \ln 3\}, \quad \ln 3 \ln ^{4} 2 .
\end{aligned}
$$

Only one extra term should be added to the weight 5 basis. This new constant can be related to a $\operatorname{LsLsc}_{1,1,3}$-function of the argument $\frac{\pi}{3}$. The results of analytical calculation of three-loop master-integrals, evaluated in [9] and in the present paper are available on

$$
\text { http : //theor.jinr.ru/ kalmykov/three-loop/master.uu }
$$

Acknowledgements. I am grateful to A. Davydychev, F. Jegerlehner, and O.V. Tarasov for useful discussions. I thank Y. Schröder and A. Vuorinen for correspondence concerning the typo in expression for the $\varepsilon$-part of $D_{4}$ and for information on the results of their calculation before publication. I would like to thank S. Mikhailov for his interest in work, and G.Sandukovskaya for careful reading of the manuscript. This research was supported in part by RFBR grant \# 04-02-17192 and the Heisenberg-Landau Programme and NATO Grant PST.CLG.980342.

\section{A Auxiliary integrals}

Below we present the values of some integrals appearing in Sec. 2.

$$
\begin{aligned}
& \int^{\theta} d \phi \frac{\cos \phi}{(\sin \phi)^{3-a \varepsilon}}=-\frac{1}{(2-a \varepsilon)} \frac{(\sin \theta)^{a \varepsilon}}{\sin ^{2} \theta} \\
& \left(1-\frac{a \varepsilon}{2}\right) \int^{\theta} d \phi \frac{\cos \phi}{\sin ^{3} \phi}(\sin \phi)^{a \varepsilon}(\cos \phi)^{b \varepsilon}=-\frac{(\sin \theta)^{a \varepsilon}(\cos \theta)^{b \varepsilon}}{2 \sin ^{2} \theta}-\frac{b \varepsilon}{2} \operatorname{Isc}_{a, b}(\theta) \\
& \left(1-\frac{a \varepsilon}{2}\right) \int^{\theta} d \phi \frac{(\sin \phi)^{a \varepsilon}(\cos \phi)^{b \varepsilon}}{\sin ^{3} \phi \cos \phi}=\left[1-\frac{(a+b) \varepsilon}{2}\right] \operatorname{Isc}_{a, b}(\theta)-\frac{(\sin \theta)^{a \varepsilon}(\cos \theta)^{b \varepsilon}}{2 \sin ^{2} \theta} \\
& (1-a \varepsilon) \int^{\theta} d \phi \frac{1}{\sin ^{2} \phi}(\sin \phi)^{a \varepsilon} \operatorname{Ls}_{j+1}(2 \phi)=-\frac{\cos \theta}{(\sin \theta)^{1-a \varepsilon}} \operatorname{Ls}_{j+1}(2 \theta) \\
& \quad-2^{-a \varepsilon} \sum_{k=0}^{\infty} \frac{(a \varepsilon)^{k}}{k !}\left[2 \frac{\ln ^{k+j+1}(2 \sin \theta)}{k+j+1}+a \varepsilon \int^{\theta} d \phi \ln ^{k}(2 \sin \phi) \operatorname{Ls}_{j+1}(2 \phi)\right] \\
& \quad+a \varepsilon) \int^{\theta} d \phi \frac{1}{\sin ^{2} \phi}(\sin \phi)^{a \varepsilon} \operatorname{Ls}_{j+1}(4 \phi)=-\frac{\cos \theta}{(\sin \theta)^{1-a \varepsilon} \operatorname{Ls}_{j+1}(4 \theta)} \\
& +2^{-1-a \varepsilon} a \varepsilon \sum_{k=0}^{\infty} \frac{(a \varepsilon)^{k}}{k !} \operatorname{Ls}_{k+1}(2 \theta) \operatorname{Ls}_{j+1}(4 \theta) \\
& +2^{1-a \varepsilon} a \varepsilon \sum_{k=0}^{\infty} \frac{(a \varepsilon)^{k}}{k !} \int^{\theta} d \phi \operatorname{Ls}_{k+1}(2 \phi)[\ln (2 \sin \phi)+\ln (2 \cos \phi)]^{j}
\end{aligned}
$$




$$
-2^{2-a \varepsilon} \sum_{k=0}^{\infty} \frac{(a \varepsilon)^{k}}{k !} \int^{\theta} d \phi \frac{\cos \phi}{\sin \phi} \ln ^{k}(2 \sin \phi)[\ln (2 \sin \phi)+\ln (2 \cos \phi)]^{j}
$$

where $\operatorname{Isc}_{a, b}(\theta)$ is defined in $(2.8)$.

\section{B Auxiliary function}

Let us describe here some properties of the new function $\operatorname{LsLsc}_{k, i, j}(\theta)$ defined as

$$
\operatorname{LsLsc}_{k, i, j}(\theta)=\int_{0}^{\theta} d \phi \operatorname{Ls}_{k+1}(\phi) \ln ^{i-1}\left|2 \sin \frac{\phi}{2}\right| \ln ^{j-1}\left|2 \cos \frac{\phi}{2}\right|,
$$

where $k, i, j$ are integer numbers, $k \geq 0$ and $i, j \geq 1$, and $\theta$ is an arbitrary real number. These functions have appeared in higher-order epsilon expansion of two- and three-loop Feynman diagrams investigated in this paper (see Eqs.(2.7) and (3.23)). LsLsc-functions are also related to the $\varepsilon$-expansion of hypergeometric functions. As was shown in [20] the multiple inverse binomial sums $\sum_{n=1}^{\infty} \frac{(n !)^{2}}{(2 n) !} \frac{z^{n}}{n^{k}} S_{1}(n-1)$ and $\sum_{n=1}^{\infty} \frac{(n !)^{2}}{(2 n) !} \frac{z^{n}}{n^{k}} S_{1}(2 n-1)$, where $S_{a}(n)$ is the harmonic sum, are expressible in terms of $\operatorname{LsLsc}_{0, i, j}(\theta), \operatorname{LsLsc}_{1, i, 1}(\theta)$ and $\operatorname{LsLsc}_{1,1, j}(\theta)$. Moreover, the elements $\chi_{5}$ and $\tilde{\chi}_{5}$ contributing to odd/even basis of a set of transcendental numbers [9] are related to $\operatorname{LsLsc}_{1,3,1}\left(\frac{2 \pi}{3}\right)$ and $\operatorname{LsLsc}_{1,3,1}\left(\frac{\pi}{2}\right)$, respectively (see Eq. (15) in $[20])$. $\operatorname{LsLsc}_{1,3,1}(\theta)$ also appears in higher order $\varepsilon$-expansion of the one-loop triangle diagram (see Eq. (4.5) in [29]). For lower values of the parameters, function (B.1) reduces to the generalized log-sine functions. In particular,

- for $k=0$ and $i=1$ or $j=1$

$$
\begin{aligned}
\operatorname{LsLsc}_{0, i, 1}(\theta) & =\operatorname{Ls}_{i+1}^{(1)}(\theta), \\
\operatorname{LsLsc}_{0,1, j}(\theta) & =\operatorname{Ls}_{j+1}^{(1)}(\pi-\theta)-\operatorname{Ls}_{j+1}^{(1)}(\pi)-\pi\left[\operatorname{Ls}_{j}(\pi-\theta)-\operatorname{Ls}_{j}(\pi)\right] .
\end{aligned}
$$

- for $k=0, i=j=1$

$$
\begin{aligned}
\operatorname{LsLsc}_{0,2,2}(\theta)= & \frac{1}{8} \operatorname{Ls}_{4}^{(1)}(2 \theta)-\frac{1}{2} \operatorname{Ls}_{4}^{(1)}(\theta)-\frac{1}{2}\left[\operatorname{Ls}_{4}^{(1)}(\pi-\theta)-\operatorname{Ls}_{4}^{(1)}(\pi)\right] \\
& +\frac{1}{2} \pi\left[\operatorname{Ls}_{3}(\pi-\theta)-\operatorname{Ls}_{3}(\pi)\right] .
\end{aligned}
$$

- for an arbitrary $k$ and $i=j=1$

$$
\operatorname{LsLsc}_{k, 1,1}(\theta)=\theta \operatorname{Ls}_{k+1}(\theta)-\operatorname{Ls}_{k+2}^{(1)}(\theta) .
$$

- for $k=1, i=2$ and $j=1$

$$
\operatorname{LsLsc}_{1,2,1}(\theta)=-\frac{1}{2}\left[\operatorname{Ls}_{2}(\theta)\right]^{2} .
$$


For $k=1, i=1$ and $j=2$ we get a function, which was denoted as $\Phi(\theta)$ in [29] (see Eq.(2.41) in [29]),

$$
\operatorname{LsLsc}_{1,1,2}(\theta)=\Phi(\theta)
$$

The relation between the functions of the opposite arguments is

$$
\operatorname{LsLsc}_{k, i, j}(-\theta)=\operatorname{LsLsc}_{k, i, j}(\theta)
$$

The symmetry relation for the new functions

$$
\operatorname{LsLsc}_{k, i, j}(\theta)-\operatorname{LsLsc}_{k, i, j}(2 \pi-\theta)+\operatorname{LsLsc}_{k, i, j}(2 \pi)=2 \operatorname{Ls}_{k+1}(\pi)\left[\operatorname{Lsc}_{i, j}(2 \pi-\theta)-\operatorname{Lsc}_{i, j}(2 \pi)\right](B .9)
$$

includes the generalized log-sine-cosine integral introduced in [9] (see Appendix A.2). Its definition is

$$
\operatorname{Lsc}_{i, j}(\theta)=-\int_{0}^{\theta} d \phi \ln ^{i-1}\left|2 \sin \frac{\phi}{2}\right| \ln ^{j-1}\left|2 \cos \frac{\phi}{2}\right| .
$$

In particular, the following relation is valid:

$$
\int_{0}^{\theta} d \phi \operatorname{Lsc}_{i, j}(\phi)=\theta \operatorname{Lsc}_{i, j}(\theta)-\operatorname{LsLsc}_{0, i, j}(\theta) .
$$

At the same time, the relation (B.9) can be considered as definition of the generalized logsine-cosine functions $\left(\operatorname{Ls}_{k}(\pi)\right.$ is a normalization constant). For $k=0$ there is an extra symmetry relation,

$$
\operatorname{LsLsc}_{0, i, j}(\theta)-\operatorname{LsLsc}_{0, j, i}(\pi-\theta)+\operatorname{LsLsc}_{0, j, i}(\pi)=-\pi\left[\operatorname{Lsc}_{j, i}(\pi-\theta)-\operatorname{Lsc}_{j, i}(\pi)\right] .
$$

When $i$ or $j$ are equal to unity, the following relations are valid:

$$
\begin{aligned}
& \operatorname{LsLsc}_{k, i, 1}(\theta)+\operatorname{LsLsc}_{i-1, k+1,1}(\theta)=-\operatorname{Ls}_{k+1}(\theta) \operatorname{Ls}_{i}(\theta) \\
& \operatorname{LsLsc}_{k, 1, j}(\theta)+\operatorname{LsLsc}_{j-1,1, k+1}(\pi-\theta)-\operatorname{LsLsc}_{j-1,1, k+1}(\pi)=\operatorname{Ls}_{k+1}(\theta) \operatorname{Ls}_{j}(\pi-\theta)
\end{aligned}
$$

The values of the function $\operatorname{LsLsc}_{k, i, j}(\theta)$ of the argument $\theta=2 \pi$ can be extracted from relation (B.9). Using the symmetric properties of the $\operatorname{Lsc}_{i, j}(\theta)$-function,

$$
\begin{aligned}
& \operatorname{Lsc}_{i, j}(-\theta)=-\operatorname{Lsc}_{i, j}(\theta) \\
& \operatorname{Lsc}_{i, j}(2 \pi-\theta)=\operatorname{Lsc}_{i, j}(2 \pi)-\operatorname{Lsc}_{i, j}(\theta) \\
& \operatorname{Lsc}_{i, j}(2 \pi)=2 \operatorname{Lsc}_{i, j}(\pi), \quad \operatorname{Lsc}_{i, j}(\pi)=\operatorname{Lsc}_{j, i}(\pi)
\end{aligned}
$$

it is easy to get

$$
\operatorname{LsLsc}_{k, i, j}(2 \pi)=-2 \operatorname{Ls}_{k+1}(\pi) \operatorname{Lsc}_{i, j}(\pi)
$$


where the values of $\operatorname{Ls}_{k+1}(\pi)$ and $\operatorname{Lsc}_{i, j}(\pi)$ can be founded in Lewin's book [21]. Taking into account that $\operatorname{Ls}_{2}(\pi)=0$ we get for $k=1$

$$
\operatorname{LsLsc}_{1, i, j}(2 \pi)=0
$$

Using relations (B.16), Eq. (B.9) can be rewritten as

$$
\operatorname{LsLsc}_{k, i, j}(\theta)-\operatorname{LsLsc}_{k, i, j}(2 \pi-\theta)=-2 \operatorname{Ls}_{k+1}(\pi)\left[\operatorname{Lsc}_{i, j}(\theta)-\operatorname{Lsc}_{i, j}(\pi)\right] .
$$

From relation (B.12) we get for $\theta=\pi$

$$
\operatorname{LsLsc}_{0, i, j}(\pi)+\operatorname{LsLsc}_{0, j, i}(\pi)=\pi \operatorname{Lsc}_{i, j}(\pi) .
$$

For particular values of the LsLsc-functions of weight 5 at $\theta=\pi, \pi / 3$ and $\theta=2 \pi / 3$ the PSLQ analysis [37] yields

$$
\begin{aligned}
& \operatorname{LsLsc}_{0,3,2}(\pi)=\frac{2}{3} \zeta_{2} \ln ^{3} 2-\frac{2}{15} \ln ^{5} 2-\frac{7}{4} \zeta_{3} \ln ^{2} 2-4 \ln 2 \operatorname{Li}_{4}\left(\frac{1}{2}\right)+\frac{155}{32} \zeta_{5}+\frac{1}{8} \zeta_{2} \zeta_{3}-4 \operatorname{Li}_{5}\left(\frac{1}{2}\right) \\
& =1.1346108755961038391083966682727 \ldots \text {, } \\
& \operatorname{LsLsc}_{1,1,3}(\pi)=\frac{2}{5} \ln ^{5} 2-2 \zeta_{2} \ln ^{3} 2+\frac{21}{4} \zeta_{3} \ln ^{2} 2+12 \ln 2 \operatorname{Li}_{4}\left(\frac{1}{2}\right)-\frac{7}{8} \zeta_{2} \zeta_{3}-\frac{155}{16} \zeta_{5}+12 \mathrm{Li}_{5}\left(\frac{1}{2}\right) \\
& =0.6301340120387385042438188494654 \ldots \text {, } \\
& \operatorname{LsLsc}_{1,3,1}(\pi)=-\frac{2}{15} \ln ^{5} 2+\frac{2}{3} \zeta_{2} \ln ^{3} 2-\frac{7}{4} \zeta_{3} \ln ^{2} 2-4 \ln 2 \mathrm{Li}_{4}\left(\frac{1}{2}\right)+\frac{155}{32} \zeta_{5}-4 \operatorname{Li}_{5}\left(\frac{1}{2}\right) \\
& =0.8874478318089418243337609880871 \ldots \text {, } \\
& \operatorname{LsLsc}_{1,2,2}(\pi)=\frac{2}{15} \ln ^{5} 2-\frac{2}{3} \zeta_{2} \ln ^{3} 2+\frac{7}{4} \zeta_{3} \ln ^{2} 2+4 \ln 2 \operatorname{Li}_{4}\left(\frac{1}{2}\right)-\frac{279}{64} \zeta_{5}+4 \operatorname{Li}_{5}\left(\frac{1}{2}\right) \\
& =-0.3851859504113720162670058305844 \ldots \text {, } \\
& \operatorname{LsLsc}_{1,3,1}\left(\frac{\pi}{3}\right)=\frac{11}{18} \zeta_{5}=0.6336780725876149549802789098028 \ldots \text {, } \\
& \operatorname{LsLsc}_{1,3,1}\left(\frac{2 \pi}{3}\right)=-\frac{55}{324} \pi \zeta_{2} \operatorname{Ls}_{2}\left(\frac{\pi}{3}\right)+\frac{1225}{1296} \zeta_{2} \zeta_{3}+\frac{4621}{1296} \zeta_{5}+\frac{23}{972} \pi \operatorname{Ls}_{4}\left(\frac{\pi}{3}\right) \\
& -\frac{5}{18} \pi \operatorname{Ls}_{4}\left(\frac{2 \pi}{3}\right)-\frac{1}{3} \operatorname{Ls}_{2}\left(\frac{\pi}{3}\right) \operatorname{Ls}_{3}\left(\frac{2 \pi}{3}\right)+\frac{1}{3} \operatorname{Ls}_{5}^{(1)}\left(\frac{2 \pi}{3}\right)-\frac{1}{48} \chi_{5} \\
& =0.7441484098381945153773320072924 \ldots \text {, } \\
& \operatorname{LsLsc}_{1,2,2}\left(\frac{\pi}{3}\right)=-\frac{55}{288} \pi \zeta_{2} \operatorname{Ls}_{2}\left(\frac{\pi}{3}\right)-\frac{3}{8} \operatorname{Ls}_{2}\left(\frac{\pi}{3}\right) \operatorname{Ls}_{3}\left(\frac{2 \pi}{3}\right)+\frac{23}{864} \pi \operatorname{Ls}_{4}\left(\frac{\pi}{3}\right) \\
& -\frac{1}{16} \pi \operatorname{Ls}_{4}\left(\frac{2 \pi}{3}\right)+\frac{361}{1152} \zeta_{2} \zeta_{3}-\frac{41}{384} \zeta_{5}-\frac{3}{128} \chi_{5} \\
& =-0.3438441037234077110928896695989 \ldots \text {, } \\
& \operatorname{LsLsc}_{1,2,2}\left(\frac{2 \pi}{3}\right)=\frac{55}{432} \pi \zeta_{2} \operatorname{Ls}_{2}\left(\frac{\pi}{3}\right)+\frac{1}{4} \operatorname{Ls}_{2}\left(\frac{\pi}{3}\right) \operatorname{Ls}_{3}\left(\frac{2 \pi}{3}\right)-\frac{23}{1296} \pi \operatorname{Ls}_{4}\left(\frac{\pi}{3}\right) \\
& +\frac{5}{24} \pi \mathrm{Ls}_{4}\left(\frac{2 \pi}{3}\right)-\frac{1225}{1728} \zeta_{2} \zeta_{3}-\frac{1379}{576} \zeta_{5}-\frac{1}{4} \mathrm{Ls}_{5}^{(1)}\left(\frac{2 \pi}{3}\right)+\frac{1}{64} \chi_{5} \\
& =-0.2676755241093223655003711724570 \ldots \text {, } \\
& \operatorname{LsLsc}_{0,3,2}\left(\frac{\pi}{3}\right)=-\operatorname{LsLsc}_{1,1,3}\left(\frac{\pi}{3}\right)+\frac{55}{216} \pi \zeta_{2} \operatorname{Ls}_{2}\left(\frac{\pi}{3}\right)+\frac{1}{2} \operatorname{Ls}_{2}\left(\frac{\pi}{3}\right) \operatorname{Ls}_{3}\left(\frac{2 \pi}{3}\right)+\frac{223}{864} \zeta_{2} \zeta_{3} \\
& -\frac{793}{864} \zeta_{5}-\frac{79}{648} \pi \operatorname{Ls}_{4}\left(\frac{\pi}{3}\right)-\frac{1}{12} \pi \operatorname{Ls}_{4}\left(\frac{2 \pi}{3}\right)+\frac{1}{4} \operatorname{Ls}_{5}^{(1)}\left(\frac{2 \pi}{3}\right)+\frac{1}{32} \chi_{5} \\
& +4 \mathrm{Li}_{5}\left(\frac{1}{2}\right)+4 \ln 2 \mathrm{Li}_{4}\left(\frac{1}{2}\right)-\frac{2}{3} \zeta_{2} \ln ^{3} 2+\frac{7}{4} \zeta_{3} \ln ^{2} 2+\frac{2}{15} \ln ^{5} 2 \\
& =-0.1728468405935728535297398287784 \ldots
\end{aligned}
$$




$$
\begin{aligned}
\operatorname{LsLsc}_{0,3,2}\left(\frac{2 \pi}{3}\right) & =\operatorname{LsLsc}_{1,1,3}\left(\frac{\pi}{3}\right)-\frac{55}{216} \pi \zeta_{2} \operatorname{Ls}_{2}\left(\frac{\pi}{3}\right)-\frac{1}{2} \operatorname{Ls}_{2}\left(\frac{\pi}{3}\right) \operatorname{Ls}_{3}\left(\frac{2 \pi}{3}\right)+\frac{1565}{864} \zeta_{2} \zeta_{3} \\
+ & \frac{7183}{864} \zeta_{5}+\frac{55}{648} \pi \operatorname{Ls}_{4}\left(\frac{\pi}{3}\right)-\frac{1}{12} \pi \operatorname{Ls}_{4}\left(\frac{2 \pi}{3}\right)-\frac{1}{2} \operatorname{Ls}_{5}^{(1)}\left(\frac{2 \pi}{3}\right)-\frac{1}{32} \chi_{5} \\
& -12 \operatorname{Li}_{5}\left(\frac{1}{2}\right)-12 \ln 2 \operatorname{Li}_{4}\left(\frac{1}{2}\right)+2 \zeta_{2} \ln ^{3} 2-\frac{21}{4} \zeta_{3} \ln ^{2} 2-\frac{2}{5} \ln ^{5} 2 \\
& =-0.2193285689662125608977695929575 \ldots \\
\operatorname{LsLsc}_{1,1,3}\left(\frac{2 \pi}{3}\right) & =-\operatorname{LsLsc}_{1,1,3}\left(\frac{\pi}{3}\right)+\frac{55}{216} \pi \zeta_{2} \operatorname{Ls}_{2}\left(\frac{\pi}{3}\right)+\frac{1}{2} \operatorname{Ls}_{2}\left(\frac{\pi}{3}\right) \operatorname{Ls}_{3}\left(\frac{2 \pi}{3}\right)-\frac{685}{864} \zeta_{2} \zeta_{3} \\
& -\frac{10235}{864} \zeta_{5}-\frac{23}{648} \pi \operatorname{Ls}_{4}\left(\frac{\pi}{3}\right)-\frac{1}{12} \pi \operatorname{Ls}_{4}\left(\frac{2 \pi}{3}\right)+\frac{1}{4} \operatorname{Ls}_{5}^{(1)}\left(\frac{2 \pi}{3}\right)+\frac{1}{32} \chi_{5} \\
+ & 16 \operatorname{Li}_{5}\left(\frac{1}{2}\right)+16 \ln 2 \operatorname{Li}_{4}\left(\frac{1}{2}\right)-\frac{8}{3} \zeta_{2} \ln ^{3} 2+7 \zeta_{3} \ln ^{2} 2+\frac{8}{15} \ln ^{5} 2 \\
& =0.4544220738685365169842148301889 \ldots
\end{aligned}
$$

where the high-precision numerical evaluation of the generalized log-sine functions was performed with the lsjk-program [39] and

$$
\operatorname{LsLsc}_{1,1,3}\left(\frac{\pi}{3}\right)=0.32403774485559909073259711644693958993 \ldots
$$

\section{References}

[1] P.A. Baikov, K.G. Chetyrkin, J.H. Kuhn, hep-ph/0412350;

K.G. Chetyrkin, J.H. Kuhn, P. Mastrolia, C. Sturm, hep-ph/0412055;

Y. Schröder, Nucl. Phys. Proc. Suppl. 116 (2003) 402.

[2] T. van Ritbergen, R.G. Stuart, Phys. Rev. Lett. 82 (1999) 488; Nucl. Phys. B 564 (2000) 343.

[3] J. Fleischer, M.Yu. Kalmykov, A.V. Kotikov, Proc. Workshop "AIHENP-99", Heraklion, Greece, April 1999 (Parisianou S.A., Athens, 2000), p. 231 (hep-ph/9905379);

J. Fleischer, M.Yu. Kalmykov, Comp. Phys. Commun. 128 (2000) 531;

http://theor.jinr.ru/ kalmykov/onshell2/onshell2.html

[4] L.V. Avdeev, Comput. Phys. Commun. 98 (1996) 15.

[5] M. Steinhauser, Comput. Phys. Commun. 134 (2001) 335.

[6] S. Laporta, E. Remiddi, Phys. Lett. B379 (1996) 283;

K. Melnikov, T. Ritbergen, Phys. Lett. B482 (2000) 99; Nucl. Phys. B591 (2000) 515.

[7] I. Blokland, A. Czarnecki, M. Slusarczyk, F. Tkachov, Phys. Rev. Lett. 93 (2004) $062001 ;$ Phys. Rev. D71 (2005) 054004.

[8] A.I. Davydychev, Proc. Workshop "AIHENP-99", Heraklion, Greece, April 1999 (Parisianou S.A., Athens, 2000), p. 219 (hep-th/9908032); Phys. Rev. D61 (2000) 087701.

[9] A.I. Davydychev, M.Yu. Kalmykov, Nucl. Phys. B605 (2001) 266. 
[10] K.S. Kölbig, J.A. Mignaco, E. Remiddi, B.I.T. 10 (1970) 38;

R. Barbieri, J.A. Mignaco, E. Remiddi, Nuovo Cim. A11 (1972) 824;

A. Devoto, D.W. Duke, Riv. Nuovo Cim. 7, No.6 (1984) 1;

K.S. Kölbig, SIAM J. Math. Anal. 17 (1986) 1232.

[11] D.J. Broadhurst, Z. Phys. C54 (1992) 599.

[12] D.J. Broadhurst, hep-th/9604128.

[13] D.J. Broadhurst, Eur. Phys. J. C8 (1999) 311.

[14] J. Fleischer, M. Yu. Kalmykov, Phys. Lett. B470 (1999) 168.

[15] K.G. Chetyrkin and M. Steinhauser, Nucl. Phys. B573 (2000) 617.

[16] M.Yu. Kalmykov, O. Veretin, Phys. Lett. B483 (2000) 315.

[17] S. Laporta, Int. J. Mod. Phys. A15 (2000) 5087; Phys. Lett. B 504 (2001) 188.

[18] S. Laporta, Phys. Lett. B549 (2002) 115;

Y. Schroder, A. Vuorinen, hep-ph/0311323; hep-ph/0503209.

[19] S. Laporta, Phys. Lett. B 523 (2001) 95.

[20] A.I. Davydychev, M.Yu. Kalmykov, Proc. of 2th CPP Symposium, Tokyo, Japan, Nov 2001 (Ed.Y.Kurihara, KEK Proceedings 2002-11), p.169 (hep-th/0203212).

[21] L. Lewin, Polylogarithms and associated functions (North-Holland, Amsterdam, 1981).

[22] G. 'tHooft and M. Veltman, Nucl. Phys. B44 (1972) 189;

C.G. Bollini and J.J. Giambiagi, Nuovo Cimento 12B (1972) 20;

J.F. Ashmore, Lett. Nuovo Cim. 4 (1972) 289;

G.M. Cicuta and E. Montaldi, Lett. Nuovo Cim. 4 (1972) 329.

[23] F. Jegerlehner, M.Yu. Kalmykov, Nucl. Phys. B676 (2004) 365.

[24] F. Jegerlehner, M.Yu. Kalmykov, Acta Phys. Polon. B34 (2003) 5335.

[25] A.V. Kotikov, Phys. Lett. B254 (1991) 158, B259 (1991) 314, B267 (1991) 123.

[26] A.I. Davydychev, R. Delbourgo, J. Math. Phys. 39 (1998) 4299.

[27] A.I. Davydychev, J.B. Tausk, Nucl. Phys. B397 (1993) 123; Phys. Rev. D 53 (1996) 7381.

[28] M. Argeri, P. Mastrolia, E. Remiddi, Nucl. Phys. B631 (2002) 388.

[29] A.I. Davydychev, M.Yu. Kalmykov, Nucl. Phys. B699 (2004) 3. 
[30] J. Fleischer, M.Yu. Kalmykov, A.V. Kotikov, Phys. Lett. B462 (1999) 169; B467 (1999) $310(\mathrm{E})$.

[31] A.I. Davydychev, M.Yu. Kalmykov, Nucl. Phys. B (Proc. Suppl.) 89 (2000) 283.

[32] E. Remiddi, J.A.M. Vermaseren, Int. J. Mod. Phys. A15 (2000) 725.

[33] M.Yu. Kalmykov, Nucl. Phys. B (Proc. Suppl.) 135 (2004) 280.

[34] V.A. Smirnov, "Applied asymptotic expansions in momenta and masses, Berlin, Germany, Springer (2002).

[35] L.V. Avdeev, J. Fleischer, M.Yu. Kalmykov, M.N. Tentyukov, Nucl. Ins. Meth. A389 (1997) 343; Comp. Phys. Commun. 107 (1997) 155; L.V. Avdeev, M.Yu. Kalmykov, Nucl. Phys. B502 (1997) 419.

[36] J.M. Chung, B.K. Chung, J. Korean Phys. Soc. 40 (2002) 435 (hep-ph/0203143).

[37] H.R.P. Ferguson, D.H. Bailey, RNR Technical Report, RNR-91-032 (1991);

H.R.P. Ferguson, D.H. Bailey, S. Arno, Math. Comput. 68 (1999) 351;

D.H. Bailey, Computing in Science \& Engineering 2, No. 1 (2000) 24.

[38] J. A. M. Vermaseren, Symbolic manipulation with FORM, Amsterdam, Computer Algebra Nederland, 1991.

[39] M.Yu. Kalmykov, A. Sheplyakov, hep-ph/0411100. 